\title{
A VERY SINGULAR SOLUTION OF A DOUBLY DEGENERATE PARABOLIC EQUATION WITH NONLINEAR CONVECTION
}

\author{
ZHONG Bo FANG
}

\begin{abstract}
We here investigate an existence and uniqueness of the nontrivial, nonnegative solution of a nonlinear ordinary differential equation:

$$
\left[\left|\left(w^{m}\right)^{\prime}\right|^{p-2}\left(w^{m}\right)^{\prime}\right]^{\prime}+\beta r w^{\prime}+\alpha w+\left(w^{q}\right)^{\prime}=0
$$

satisfying a specific decay rate: $\lim _{r \rightarrow \infty} r^{\alpha / \beta} w(r)=0$ with $\alpha:=(p-$ $1) /[p q-(m+1)(p-1)]$ and $\beta:=[q-m(p-1)] /[p q-(m+1)(p-1)]$. Here $m(p-1)>1$ and $m(p-1)<q<(m+1)(p-1)$. Such a solution arises naturally when we study a very singular solution for a doubly degenerate equation with nonlinear convection:

$$
u_{t}=\left[\left|\left(u^{m}\right)_{x}\right|^{p-2}\left(u^{m}\right)_{x}\right]_{x}+\left(u^{q}\right)_{x}
$$
\end{abstract}

defined on the half line.

\section{Introduction}

In this paper, we consider a one dimensional doubly degenerate equation with nonlinear convection term

$$
u_{t}=\left[\left|\left(u^{m}\right)_{x}\right|^{p-2}\left(u^{m}\right)_{x}\right]_{x}+\left(u^{q}\right)_{x}, \quad(x, t) \in \mathbb{R}^{+} \times \mathbb{R}^{+}
$$

with Neumann boundary condition

$$
u_{x}(0, t)=0,
$$

where $m(p-1)>1, q>m(p-1)$.

Equation (1.1) (sometimes called the non-Newtonian filtration equation) arises in the study a compressible fluid flows in a homogeneous isotropic rigid porous medium, flows of polytrophic gas and has various other applications, see, [23], [5]. From a mathematical point of view, we note that (1.1) is a quasilinear equation which is nonuniform parabolic and it is doubly degenerate on

Received September 30, 2008.

2000 Mathematics Subject Classification. 60G18, 35K65, 35B40, 34A12.

Key words and phrases. very singular solution, existence, uniqueness, asymptotic behavior.

The Project-sponsored by SRF for ROCS, SEM. 
the sets $\left\{u_{x}=0\right\}$ and $\{u=0\}$ (if $q=1$, equation (1.1) reduces to the standard doubly degenerate equation by an easy change of variables). The case $m(p-1)>1$ occurs in slow diffusion process and $0<m(p-1)<1$ in fast diffusion process (see [19] and [22] for examples).

We are mostly interested in nonnegative solutions of (1.1) having the form

$$
u(x, t)=t^{-\alpha} w\left(x t^{-\beta}\right):=t^{-\alpha} w(r),
$$

where $\alpha, \beta$ are positive numbers. We substitute (1.3) into (1.1) to find

(1.4) $\alpha:=(p-1) /[p q-(m+1)(p-1)], \quad \beta:=[q-m(p-1)] /[p q-(m+1)(p-1)]$ and $w$, as a function of $r=x t^{-\beta}$, solves an ordinary differential equation:

$$
\left[\left|\left(w^{m}\right)^{\prime}\right|^{p-2}\left(w^{m}\right)^{\prime}\right]^{\prime}+\beta r w^{\prime}+\alpha w+\left(w^{q}\right)^{\prime}=0 .
$$

We observe that if $u(x, t)$ solves (1.1), then the rescaled functions

$$
u_{\rho}(x, t)=\rho^{(p-1) /[q-m(p-1)]} u\left(\rho x, \rho^{[p q-(m+1)(p-1)] /[q-m(p-1)]} t\right), \quad \rho>0
$$

define a one parameter family of solutions to (1.1). A solution $u(x, t)$ is said to be self-similar when $u_{\rho}(x, t)=u(x, t)$ for every $\rho>0$. It can be easily verified that $u(x, t)$ is a self-similar solution to (1.1) if and only if $u$ has the form (1.3). We also remark that the self-similar solutions play an important role in the study of large time behaviors of general solutions (see [16, 18] and [24]).

Every nonnegative, bounded solution of (1.5) has exactly one critical point and since we here apply the shooting method, led to solve a more general initial value problem

$$
\left[\left|\left(w^{m}\right)^{\prime}\right|^{p-2}\left(w^{m}\right)^{\prime}\right]^{\prime}+\beta r w^{\prime}+\alpha w+\left(w^{q}\right)^{\prime}=0
$$

for $r \geq 0$ with initial conditions

$$
w^{\prime}(0)=0, \quad w(0)=\mu,
$$

where $\mu$ may be any positive number.

Using the Shauder's fixed point theorem (or Banach contraction theorem), we find that initial value problem has an unique solution which we denote by $w(r ; \mu)$. In many cases, it turns out that the limit

$$
L(\lambda)=\lim _{r \rightarrow \infty} r^{\alpha / \beta} w(r)
$$

exists and we distinguish between fast and slow orbits according to whether $L(\lambda)=0$ or not respectively. The fast orbit will bring out a very singular solution of (1.1). The very singular solution has a stronger singularity at the origin than the singular solution of that equation. By a singular solution we mean a nonnegative and nontrivial solution which satisfies the equation and vanishes outside any open neighborhood of the origin as $t \rightarrow 0$. A singular solution is called a very singular solution if the integral of $u(x, t)$ over any open neighborhood of the origin becomes unbounded as $t \rightarrow 0$, which is equivalent to, if $u$ is given by (1.3),

$$
\lim _{r \rightarrow \infty} r^{\alpha / \beta} w(r)=0
$$


Furthermore, if $0<\beta<\alpha$ and a solution $f$ of (1.5) satisfies (1.9), then $u(x, t)$ given explicitly by (1.3) becomes a very singular self-similar solution of (1.1).

Our goal is to find the relation of values $m, p, q$ and initial data $\mu$ which insure that $w(\cdot, \mu)$ is a fast decaying solution and to give an exact asymptotic behavior of solutions at near infinity. More precisely, our main results include the followings;

- If $\alpha \leq \beta$ (i.e., $q \geq(m+1)(p-1))$, then there not exists any fast orbit (very singular solution) and indeed, only exists slow orbits for any $\mu>0$.

- If $\alpha>\beta$ (i.e., $m(p-1)<q<(m+1)(p-1))$, then there exists $\mu_{1}$ such that

(i) $w(r ; \mu)$ is changes sign with $w^{m}\left(R^{-} ; \mu\right)<0$ for $\mu \in\left(0, \mu_{1}\right)$.

(ii) $w(r ; \mu)$ is a slow orbit and having the behavior

$$
w(r ; \mu) \sim L(\mu) r^{-\alpha / \beta}
$$

at near of infinity for $\mu \in\left(\mu_{1},+\infty\right)$, with $L(\mu)>0$.

(iii) $w\left(r ; \mu_{1}\right)$ is the only fast orbit with $w^{m}\left(R^{-} ; \mu\right)=0$ and having the interface relation

$$
\lim _{r \rightarrow R^{-}}\left(w^{[m(p-1)-1] /(p-1)}\right)^{\prime}(r)=-[m(p-1)-1] /[m(p-1)] \beta^{1 /(p-1)} R^{1 /(p-1)}
$$

for some $0<R<\infty$.

There have been many works dealing with the existence, uniqueness and asymptotic behavior of self-similar solutions to a class of quasilinear parabolic equations with absorption (or source, convection) term. For instance, it is thoroughly treated on the P-Laplacian equation with absorption term;

$$
u_{t}=\operatorname{div}\left(|\nabla u|^{p-2} \nabla u\right)-u^{q} \quad \text { in } \mathbb{R}^{\mathbb{N}} \times \mathbb{R}^{+}
$$

with $p>1, q>1$. For linear diffusion case $(p=2)$, see [3], [6], [13] for slow diffusion case $(p>2)$, see [22] and [1], [16] for fast diffusion case $(1<p<2)$.

Recently some authors studied (1.1) with $m(p-1) \geq 1, q>m(p-1)$. They derived some estimates, used a suitable scaling and convergence of re-scaled solutions to self-similar ones, and concluded that the asymptotic of general solutions is self-similar (see $[16,17,18,24]$ ). Similar arguments have been used in the case of the multidimensional convection-diffusion equation (see $[7,8]$, for examples). In addition, very singular self-similar solutions are found for the linear diffusion equation with convection on half line under the homogeneous Neumann boundary condition which motivated our investigation (see [2], [11], and $[20])$. 
Let $f=w^{m}, \lambda=\mu^{m}$. Then the initial value problem (1.5), (1.7) is replaced by the following problem with respect to $f$

$$
\left\{\begin{array}{l}
\left(\left|f^{\prime}\right|^{p-2} f^{\prime}\right)^{\prime}+\beta r\left(f^{1 / m}\right)^{\prime}+\alpha f^{1 / m}+\left(f^{q / m}\right)^{\prime}=0 \text { in } r>0 \\
f(r)>0 \\
f^{\prime}(0)=0, \quad f(0)=\lambda>0
\end{array}\right.
$$

and the condition (1.9) is replaced by

$$
\lim _{r \rightarrow \infty} r^{\alpha / \beta} f^{1 / m}(r ; \lambda)=0 .
$$

Indeed, all throughout this paper we consider the above problem with respect to $f$ and always assume that $[0, R)$ is the maximal existence interval of nonnegative solution of $w$ or $f$.

The plan of this paper is as follows. In Section 2, we study basic properties of $f$ which will be useful in the proof of the main results. In Section 3, we study the nonexistence of the very singular solution(fast orbit) when $q \geq(m+1)(p-$ 1). In Section 4, we study the existence changing sign solutions, fast(slow) decaying global solutions and finding the decay rates, the interface relation when $m(p-1)<q<(m+1)(p-1)$. In Section 5, we show that uniqueness of the very singular solution.

\section{Preliminary results}

In this section we shall derive some properties of $f$ which will be useful in the proof of the main results.

We first show that the sign of $f^{\prime}$ are depending on the sign of $\alpha$ and $f$ decreases as long as it is positive, and also give the behavior of $f, f^{\prime}$ at near of infinity.

Lemma 2.1. Assume that $\alpha>0, \beta>0$ and $\lambda>0$. Let $f$ be a solutions to (1.10) such that $f>0$ on $[0, R)$ with $R$ possibly infinity. Then

(i) $\lim _{r \rightarrow R^{-}} f(r)=0$.

(ii) $f^{\prime}(r)<0$ in $(0, R)$.

(iii) $\lim _{r \rightarrow \infty} f^{\prime}(r)=0$ when $R=\infty$.

Proof. We first to show that (ii).

By (1.10) we obtain $\left(\left|f^{\prime}\right|^{p-2} f^{\prime}\right)^{\prime}(0)=-\alpha \lambda^{1 / m}<0$. Thus, the function is strictly decreasing for small $r$. Suppose that there exists first zero of $f^{\prime}$ is $r_{1}$ such that $f(r)>0$ on $\left(0, r_{1}\right)$ and $f^{\prime}\left(r_{1}\right)=0$. From $(1.10)$ one sees $\left(\left|f^{\prime}\right|^{p-2} f^{\prime}\right)^{\prime}\left(r_{1}\right)<$ 0 , which is impossible.

Since $f$ is strictly decreasing and $f$ is bounded below by 0 , there exists

$$
\lim _{r \rightarrow R^{-}} f(r)=l \in[0, \lambda) .
$$

We define the energy function $E(r)=(p-1) / p\left|f^{\prime}\right|^{p}+m \alpha /(m+1) f^{(m+1) / m}$ and obtain

$$
\frac{d}{d r} E(r)=-\left(f^{\prime}\right)^{2} / m\left(\beta r f^{(1-m) / m}+q f^{(q-m) / m}\right)<0
$$


for $r>0$. Thus, $E(r)$ decreases monotonically to a limit and there also exists the limit

$$
\lim _{r \rightarrow R^{-}} f^{\prime}(r)=-l_{1}, \quad l_{1} \in[0, \infty) .
$$

In particular $l_{1}$ must be zero so that $f$ is positive for all positive $r$.

Next, we prove that $l=0$. Suppose to the contrary $l>0$. By (iii) we obtain

$$
\liminf _{r \longrightarrow \infty}\left|f^{\prime \prime}(r)\right|=0 .
$$

Moreover, we easy to see that

$$
\lim _{r \longrightarrow \infty} r\left(f^{1 / m}\right)^{\prime}(r)=-\alpha / \beta l^{1 / m}<0 .
$$

Indeed, the function $r\left(f^{1 / m}\right)^{\prime}$ is either eventually oscillates or monotone. If monotone, clearly holds by (1.5), (2.3) and if oscillates, we choose the sequence $r_{j}$ realizing the minima(or maxima) of the function $r\left(f^{1 / m}\right)^{\prime}$, then remain holds above result (2.4).

By (2.4) yields there exists $r_{0}$ such that

$$
\left(f^{1 / m}\right)^{\prime}<-C / r \text { for } r \geq r_{0},
$$

where $C>0$, which implies that $f(r) \rightarrow-\infty$ as $r \rightarrow+\infty$, which leads to a contradiction.

By Lemma 2.1 (ii), $f^{\prime}(r)<0$ in $(0, R)$ for any $\lambda>0$ and we find that if $R<\infty$, then $f(R)=0$ and $f^{\prime}(R) \leq 0$. We next show that if $f^{\prime}(R)=0$, then $f$ vanishes identically after $R$.

Lemma 2.2. Assume that $\alpha>0$ and $\lambda>0$. Let $f$ be any solution of (1.10) with $f(R)=f^{\prime}(R)=0$ for $R>0$. Then $f=0$ for all $r \geq R$.

Proof. By convention, (1.10) is rewritten as

$$
\left(\left|f^{\prime}\right|^{p-2} f^{\prime}\right)^{\prime}+\beta r\left(f^{1 / m}\right)^{\prime}+\alpha f^{1 / m}+\left(|f|^{(q-m) / m} f\right)^{\prime}=0 .
$$

Thus, without loss of generality, we may assume that $f(r)>0$ and $f^{\prime}(r)>0$ for $r$ near $R$ with $r>R$. For such $r$, we find easily from $(2.5)$ that $\left(\left|f^{\prime}\right|^{p-2} f^{\prime}\right)^{\prime}(r)<$ 0 . Integrating over $(R, r)$, we see that $\left|f^{\prime}\right|^{p-2} f^{\prime}(r)<0$, which contradict to the assumption.

\section{The case $\beta \geq \alpha(q \geq 2(p-1))$}

In this section, we show that there does not exist any fast orbit for the problem (1.10) and thus no very singular solution for (1.1) when $0<\alpha \leq \beta$.

Theorem 3.1. Assume $\beta \geq \alpha(q \geq(m+1)(p-1))$. For each $\lambda>0$, let $f(r ; \lambda)$ be the solution of (1.10). Then $R=\infty$ and $\liminf _{r \rightarrow \infty} r^{\alpha / \beta} f^{1 / m}(r ; \lambda)>0$. 
Proof. We assume $R<\infty$ to the contrary and integrate $(1.10)$ over $(0, R)$ to get

$$
\left|f^{\prime}\right|^{p-2} f^{\prime}(R)+(\alpha-\beta) \int_{0}^{R} f^{1 / m}(r) d r-\lambda^{q / m}=0,
$$

which is impossible. Thus $f$ is positive for all $r \geq 0$ and $R=\infty$.

Moreover, we have, for $r>0$,

$$
\begin{aligned}
& \left\{r^{\alpha / \beta-1}\left|f^{\prime}\right|^{p-2} f^{\prime}+\beta r^{\alpha / \beta} f^{1 / m}\right\}^{\prime} \\
= & r^{\alpha / \beta-1}\left\{\left(\left|f^{\prime}\right|^{p-2} f^{\prime}\right)^{\prime}+\frac{\alpha / \beta-1}{r}\left|f^{\prime}\right|^{p-2} f^{\prime}+\alpha f^{1 / m}+\beta r\left(f^{1 / m}\right)^{\prime}\right\} .
\end{aligned}
$$

By (1.5), we get

$$
\left\{r^{\alpha / \beta-1}\left|f^{\prime}\right|^{p-2} f^{\prime}+\beta r^{\alpha / \beta} f^{1 / m}\right\}^{\prime}=r^{\alpha / \beta-1}\left\{\frac{\alpha / \beta-1}{r}\left|f^{\prime}\right|^{p-2} f^{\prime}-\left(f^{q / m}\right)^{\prime}\right\}>0
$$

by the condition $\beta \geq \alpha$ and $f^{\prime}<0$. If we define the function

$$
F(r):=r^{\alpha / \beta-1}\left|f^{\prime}\right|^{p-2} f^{\prime}+\beta r^{\alpha / \beta} f^{1 / m},
$$

then we see that $F(0)=0$ and $F(r)$ is strictly increasing for all $r>0$. Since $f$ is a decreasing function, one must have $\liminf _{r \rightarrow \infty} r^{\alpha / \beta} f^{1 / m}(r ; \lambda)>0$.

We will see later that the $\operatorname{limit}_{\lim _{r \rightarrow \infty}} r^{\alpha / \beta} f^{1 / m}(r ; \lambda)$ exists for each $\lambda>0$. Thus we may conclude together with Theorem 3.1 that there exist slow orbits only.

$$
\text { 4. The case } \alpha>\beta(m(p-1)<q<(m+1)(p-1))
$$

In this section, we first show that the solution changes sign for small $\lambda$ and we next show that the solution becomes a slow orbit for suitably large $\lambda$. We then find a fast orbit in-between. The slow orbits will be shown to be ordered and the minimal one becomes the fast orbit as we have seen in many cases, see [10], [13], [1], [19], [20] for examples.

Define the following three sets for any initial value $\lambda>0$,

$$
\begin{aligned}
& \mathcal{S}_{1}=\left\{\lambda>0 ; R<\infty, f^{\prime}\left(R^{-}, \lambda\right)<0\right\}, \\
& \mathcal{S}_{2}=\left\{\lambda>0 ; R<\infty, f^{\prime}\left(R^{-}, \lambda\right)=0\right\}, \\
& \mathcal{S}_{3}=\{\lambda>0 ; R=\infty, f(r, \lambda)>0\} .
\end{aligned}
$$

Obviously, there sets are disjoint and $\mathcal{S}_{1} \cup \mathcal{S}_{2} \cup \mathcal{S}_{3}=(0, \infty)$.

We first show that the problem (1.10) has changes sign for "small" $\lambda>0$.

Theorem 4.1. The set $\mathcal{S}_{1} \neq \emptyset$ and open.

Proof. By integrating (1.10), one has

$$
\left|f^{\prime}\right|^{p-2} f^{\prime}+\beta r f^{1 / m}=\phi(r):=-(\alpha-\beta) \int_{0}^{r} f^{1 / m} d r-f^{q / m}+\lambda^{q / m} .
$$

One finds easily that $\phi(0)=0, \phi^{\prime}(r)=-(\alpha-\beta) f^{1 / m}-q / m f^{(q-m) / m} f^{\prime}$, and $\phi^{\prime}(0)=-(\alpha-\beta) \lambda<0$. 
Suppose that $\phi(r)<0$ and thus

$$
\left|f^{\prime}\right|^{p-2} f^{\prime}+\beta r f^{1 / m}<0, \quad 0<r<r_{0}
$$

for some $r_{0}$ to be determined later. An integration of (4.2) yields

$$
f^{\frac{m(p-1)-1}{m(p-1)}}(r)<\lambda^{\frac{m(p-1)-1}{m(p-1)}}-\frac{m(p-1)^{2} \beta^{1 /(p-1)}}{p[m(p-1)-1]} r^{p /(p-1)} .
$$

Thus if $r_{0}>R_{0}:=\left(\frac{p[m(p-1)-1]}{m(p-1)^{2} \beta^{1 /(p-1)}} \lambda^{\frac{m(p-1)-1}{m(p-1)}}\right)^{(p-1) / p}$, then $f$ must change sign and we are done. Otherwise, we may assume that $\phi\left(r_{0}\right)=0$ for some $r_{0} \leq R_{0}$. From definition, we obtain $f^{\prime}\left(r_{0}\right)=-\beta^{1 /(p-1)} r_{0}^{1 /(p-1)} f^{1 /[m(p-1)]}\left(r_{0}\right)$ and

$$
\phi^{\prime}\left(r_{0}\right)=-(\alpha-\beta) f^{1 / m}\left(r_{0}\right)-q / m f^{q / m-1} f^{\prime}\left(r_{0}\right) \geq 0 .
$$

Combining these, we have

$$
0<\alpha-\beta \leq q / m \beta^{1 /(p-1)} r_{0}^{1 /(p-1)} f^{q / m-[(m+1)(p-1)-1] /[m(p-1)]} .
$$

Since $f$ is a decreasing solution, we also have $f\left(r_{0}\right) \leq \lambda$ and

$$
\begin{aligned}
\alpha-\beta \leq & q / m \beta^{1 /(p-1)}\left(\frac{p[m(p-1)-1]}{m(p-1)^{2} \beta^{1 /(p-1)}}\right)^{1 / p} \\
& \cdot \lambda^{\frac{m(p-1)-1}{m p}+q / m-[(m+1)(p-1)-1] /[m(p-1)]} .
\end{aligned}
$$

The inequality (4.3) does not hold for all sufficiently small $\lambda$, which proves the first part of theorem. The continuous dependence of solutions on the initial values implies that $\mathcal{S}_{1}$ is an open set.

We next prove that the problem (1.10) has a global positive decaying solution for all suitably large $\lambda$.

Lemma 4.2. Let $\alpha>\beta$. Then for any $R_{0}$ there exists $\lambda_{0}$ such that $f(r)=$ $f(r, \lambda)>0$ for $0<r<R_{0}$ and $f\left(R_{0}\right)+\left|f^{\prime}\left(R_{0}\right)\right|^{p-2} f^{\prime}\left(R_{0}\right)>0$ for all $\lambda \geq \lambda_{0}$.

Proof. We define $f_{\lambda}(t)=\frac{1}{\lambda} f(r, \lambda), t=r \lambda^{\delta}$ with $\delta=\frac{[q-m(p+1)]}{m(p-1)}$. Then $f_{\lambda}$ satisfies $f_{\lambda}^{\prime}(0)=0, f_{\lambda}(0)=1$ and the following equation

$$
\left(\left|f_{\lambda}^{\prime}\right|^{p-2} f_{\lambda}^{\prime}\right)^{\prime}+\lambda^{-\frac{q p-(m+1)(p-1)}{m(p-1)}}\left[\beta t\left(f_{\lambda}^{1 / m}\right)^{\prime}+\alpha f_{\lambda}^{1 / m}\right]+\left(f_{\lambda}^{q / m}\right)^{\prime}=0 .
$$

By integrating over $(0, t)$, we obtain

$$
\begin{aligned}
& \left|f_{\lambda}^{\prime}\right|^{p-2} f_{\lambda}^{\prime}+\lambda^{-\frac{q p-(m+1)(p-1)}{m(p-1)}}(\alpha-\beta) \\
& \cdot \int_{0}^{t} f_{\lambda}^{1 / m} d \tau+\lambda^{-\frac{q p-(m+1)(p-1)}{m(p-1)}} \beta t f_{\lambda}^{1 / m}+\left(f_{\lambda}^{q / m}-1\right)=0 .
\end{aligned}
$$

Since $f_{\lambda}$ is bounded by 1 , for any $\epsilon>0$ there is $\lambda_{0}$ such that whenever $\lambda \geq \lambda_{0}$,

$$
1-\epsilon<\left|f_{\lambda}^{\prime}\right|^{p-2} f_{\lambda}^{\prime}+f_{\lambda}^{q / m}<1+\epsilon
$$

for $t \in\left[0, \frac{q p-(m+1)(p-1)}{m(p-1)}-\epsilon\right]$, which implies lemma. 
We also prove the next key-observation:

Proposition 4.3. Assume that $\alpha>0, \beta>0$ and $\lambda>0$. Let $f$ be any solution to (1.10). Consider the function $E_{c}(r):=c f+r f^{\prime}$ for $c>0$. Then

(i) If $c>m \alpha / \beta$, then $E_{c}(r)$ is eventually positive.

(ii) If $c<m \alpha / \beta$, then $E_{c}(r)$ is eventually negative.

Proof. By direct calculations and (1.10), we obtain

$$
\begin{aligned}
& (p-1)\left|f^{\prime}\right|^{p-2} E_{c}^{\prime}(r) \\
= & (c+1)(p-1)\left|f^{\prime}\right|^{p-2} f^{\prime}-\beta r^{2}\left(f^{1 / m}\right)^{\prime}-\alpha r f^{1 / m}-q / m r f^{(q-m) / m} f^{\prime}
\end{aligned}
$$

and at any $r=r_{0}$ for which $E_{c}\left(r_{0}\right)=0$ we have

$$
\begin{aligned}
& (p-1)\left|f^{\prime}\right|^{p-2} E_{c}^{\prime}\left(r_{0}\right) \\
= & -(c+1)(p-1) c^{p-1}\left(f / r_{0}\right)^{p-1}+(\beta / m c-\alpha) r_{0} f^{1 / m}+q c / m f^{q / m} .
\end{aligned}
$$

Since the middle term on the right hand side of (4.5) dominates the others for all sufficiently large $r_{0}$, the sign of $E_{c}^{\prime}\left(r_{0}\right)$ is only decided by the sign of $\beta / m c-\alpha$ and thus $E_{c}(r)$ becomes of the same sign eventually.

In order prove (i), we suppose that there exists $r_{1}$ such that $E_{c}(r)<0$ for all $r \geq r_{1}$. From equation (1.10) and Lemma 2.1 (ii) we deduce that

$$
\left(\left|f^{\prime}\right|^{p-2} f^{\prime}\right)^{\prime}-(\beta / m c-\alpha) f^{1 / m}=-\beta / m f^{1 / m-1} E_{c}(r)-\left(f^{q / m}\right)^{\prime}>0
$$

for $r \geq r_{1}$. Multiplying the previous inequality by $f^{\prime}$ and integrating from $r$ to $\tau$ with $r_{1} \leq r \leq \tau$, we have

$$
(p-1) / p\left|f^{\prime}\right|^{p}(\tau)-c_{1} f^{(m+1) / m}(\tau) \leq(p-1) / p\left|f^{\prime}\right|^{p}(r)-c_{1} f^{(m+1) / m}(r),
$$

where $c_{1}:=(\beta c-m \alpha) /(m+1)$. Letting $\tau \rightarrow \infty$ and using Lemma 2.1(ii), (iii), we get the following inequality

$$
-f^{\prime} f^{-\frac{m+1}{m p}} \geq c_{2}>0, \quad r \geq r_{1} .
$$

Integrating the previous inequality from $r_{1}$ to $r \geq r_{1}$ we obtain

$$
\begin{aligned}
& m p /[m(p-1)-1] f^{[m(p-1)-1] /(m p)}\left(r_{1}\right) \\
& \quad-m p /[m(p-1)-1] f^{[m(p-1)-1] /(m p)}(r) \geq c_{2}\left(r-r_{1}\right) .
\end{aligned}
$$

Letting $r \rightarrow \infty$ we get a contradiction.

We prove (ii) similarly. Suppose that there exists $r_{2}$ such that $E_{c}(r)>0$ for all $r \geq r_{2}$. From (1.10) and assumption,

$$
\begin{aligned}
\left(\left|f^{\prime}\right|^{p-2} f^{\prime}\right)^{\prime}+\alpha f^{1 / m} & =-\beta r\left(f^{1 / m}\right)^{\prime}+\alpha f^{1 / m}-q / m f^{q / m-1} f^{\prime} \\
& \leq \beta / m c f^{1 / m}+q / m c / r f^{q / m}
\end{aligned}
$$

Since $f$ decrease, we may rewrite this as

$$
\left(\left|f^{\prime}\right|^{p-2} f^{\prime}\right)^{\prime} \leq-c_{2} f<c_{2} \frac{r f^{\prime}}{c},
$$


where we define $c_{2}=\alpha \lambda^{1 / m-1}-c \beta / m \lambda^{1 / m-1}+\frac{c q}{m r_{2}} \lambda^{q / m-1}$ and assume to be positive by retaking $r_{2}$. The inequality (4.6) is rewritten as $(p-1) /(p-$ 2) $\left(\left|f^{\prime}\right|^{p-2} f^{\prime}\right)^{\prime} \leq-c_{3} r$ for some positive constant $c_{3}$ and an integration from $r=r_{2}$ to $r=\infty$ yields a contradiction, which completes the proof.

We rewrite the problem (1.10) as the following system;

$$
\left\{\begin{array}{l}
f^{\prime}=|h|^{-(p-2) /(p-1)} h \\
h^{\prime}=-\beta / m r f^{1 / m-1}|h|^{-(p-2) /(p-1)} h \\
\quad-\alpha f^{1 / m}-q / m f^{(q-m) / m}|h|^{-(p-2) /(p-1)} h .
\end{array}\right.
$$

Given any $\delta>0$, we denote

$$
\mathcal{L}_{\delta}:=\{(f, h): 0<f \leq 1,0>h>-\delta f\}
$$

then we obtain the following lemma.

Lemma 4.4. For given $\delta>0$ there exists a $r_{\delta}:=m\left[\delta+\alpha \delta^{-1 /(p-1)}\right] / \beta$ such that $\mathcal{L}_{\delta}$ is positively invariant for $r>t_{\delta}$. That is $\left(f\left(r_{\delta}\right), h\left(r_{\delta}\right)\right) \in \mathcal{L}_{\delta}$ then the orbit $(f(r), h(r))$ of $(4.7)$ remains in region $\mathcal{L}_{\delta}$ for all $r \geq r_{\delta}$.

Proof. We shall show that given $\delta>0$ there exists a $r_{\delta}>0$ such that if $r>r_{\delta}$, then the vector field determined by $(4.7)$ points into $\mathcal{L}_{\delta}$, except at the critical point (0. 0$)$. It is easy to see this fact on the top $h=0$ and the line $f=1$ and it is enough to verify this only on the line $h=-\delta f$. By the system (4.7), we have

$$
\begin{aligned}
& \frac{h^{\prime}}{f^{\prime}} \\
= & \frac{-\beta / m r f^{1 / m-1}|h|^{-(p-2) /(p-1)} h-\alpha f^{1 / m}-q / m f^{(q-m) / m}|h|^{-(p-2) /(p-1)} h}{|h|^{-(p-2) /(p-1)} h} \\
= & -\beta / m r f^{1 / m-1}+\alpha \delta^{-1 /(p-1)} f^{[(p-1)-m] /[m(p-1)]}-q / m f^{(q-m) / m} \\
< & -\beta / m r f^{1 / m-1}+\alpha \delta^{-1 /(p-1)} f^{[(p-1)-m] /[m(p-1)]} \leq-\delta \\
\text { if } r & \geq r_{\delta}:=m\left[\delta+\alpha \delta^{-1 /(p-1)}\right] / \beta .
\end{aligned}
$$

As a consequence, we can prove the existence of globally positive solutions.

Theorem 4.5. The set $\mathcal{S}_{3}$ is nonempty and open.

Proof. From Lemma 4.2, we can find $r_{0}$ such that $f>0$ for $0 \leq r \leq r_{0}$ and $f\left(r_{0}\right)+\left|f^{\prime}\left(r_{0}\right)\right|^{p-2} f^{\prime}\left(r_{0}\right)>0$ for all sufficiently large $\lambda$. Thus $\left(f\left(r_{0}\right)\right.$, $\left.\left|f^{\prime}\right|^{p-2} f^{\prime}\left(r_{0}\right)\right) \in \mathcal{L}_{1}$ and by Lemma $4.4, f$ is positive for all $r>0$, which proves the first part of theorem.

We next prove $\mathcal{S}_{3}$ is an open set. Set $\lambda_{0} \in \mathcal{S}_{3}$ and then by Proposition 4.3, $E_{1}(r)=f+r f^{\prime}$ becomes positive for all large $r$. Thus there exist sufficiently large $r_{0}$ such that $\left(f\left(r_{0}\right),\left|f^{\prime}\right|^{p-2} f^{\prime}\left(r_{0}\right)\right) \in \mathcal{L}_{1}$. Then by continuous dependence of solutions on the initial value there is a neighborhood $N$ of $\lambda_{0}$ such that $f(r ; \lambda)>0$ and $\left(f\left(r_{0} ; \lambda\right),\left|f^{\prime}\right|^{p-2} f^{\prime}\left(r_{0} ; \lambda\right)\right) \in \mathcal{L}_{1}$ for any $(r, \lambda) \in\left[0, r_{0}\right] \times N$. By 
Lemma 4.4, we deduce that the orbits remains in $\mathcal{L}_{1}$ for any $r>r_{0}$, which implies in particular that $f(r, \lambda)>0$ for any $r>r_{0}$ and $\lambda \in N$. Therefore, $f(r ; \lambda)>0$ for any $r>0$ and $\lambda \in N$ and $\mathcal{S}_{3}$ is open.

We are now going to find exact decay-rates for globally positive solutions.

Theorem 4.6. For any given $\lambda>0$, let $f$ be any solution to (1.10) such that $f>0$ for any $r>0$. Then $\lim _{r \rightarrow \infty} r^{\alpha / \beta} f^{1 / m}(r ; \lambda)=\mathrm{L}(\lambda)>0$ exists.

Proof. Step 1: By Lemma 2.1 we know that $f^{\prime}(r)<0$ for $r>0$ and $\lim _{r \rightarrow \infty} f(r)=0, \lim _{r \rightarrow \infty} f^{\prime}(r)=0$. Moreover we have seen that if $c<m \alpha / \beta$, then $E_{c}(r)=c f+r f^{\prime}<0$ for all sufficiently large $r$, say, $r>r_{0}$. We easily find that

$$
f(r) \leq f\left(r_{0}\right) r^{-c}, \quad r>r_{0} .
$$

We also recall that if $d>m \alpha / \beta$, then $E_{d}(r)=d f+r f^{\prime}>0$ and thus

$$
-f^{\prime}(r)<d f(r) / r, \quad r>r_{1}
$$

for some $r_{1}>0$.

Step 2: From (1.10), we get

$$
\begin{aligned}
& \left\{r^{\alpha / \beta-1}\left|f^{\prime}\right|^{p-2} f^{\prime}+\beta r^{\alpha / \beta} f^{1 / m}\right\}^{\prime} \\
= & r^{\alpha / \beta-1}\left\{\frac{\alpha / \beta-1}{r}\left|f^{\prime}\right|^{p-2} f^{\prime}-\left(f^{q / m}\right)^{\prime}\right\},
\end{aligned}
$$

and integrating over $(0, r)$, we see that

$$
\begin{aligned}
& r^{\alpha / \beta-1}\left|f^{\prime}\right|^{p-2} f^{\prime}+\beta r^{\alpha / \beta} f^{1 / m} \\
= & (\alpha / \beta-1) \int_{0}^{r}\left|f^{\prime}\right|^{p-2} f^{\prime} s^{\alpha / \beta-2} d s+q / m \int_{0}^{r} f^{(q-m) / m}\left|f^{\prime}\right| s^{\alpha / \beta-1} d s .
\end{aligned}
$$

Using (4.8) and (4.9), we find that two integrals of the right hand side of (4.11) converge and $\lim _{r \rightarrow \infty} r^{\alpha / \beta-1}\left|f^{\prime}\right|^{p-2} f^{\prime}=0$. Therefore, the limit $\mathrm{L}(\lambda)=$ $\lim _{r \rightarrow \infty} r^{\alpha / \beta} f^{1 / m}(r ; \lambda)$ exists and finite.

Step 3: We now show that $\mathrm{L}(\lambda)>0$. Assume that $\mathrm{L}(\lambda)=0$. Integrating (4.10) over $(r, \infty)$, we have

$$
\begin{aligned}
& r^{\alpha / \beta-1}\left|f^{\prime}\right|^{p-2} f^{\prime}+\beta r^{\alpha / \beta} f^{1 / m} \\
= & (1-\alpha / \beta) \int_{r}^{\infty}\left|f^{\prime}\right|^{p-2} f^{\prime} s^{\alpha / \beta-2} d s-q / m \int_{r}^{\infty} f^{(q-m) / m}\left|f^{\prime}\right| s^{\alpha / \beta-1} d s .
\end{aligned}
$$

Again using (4.9), we see that $\mathrm{L}(\lambda)=\lim _{r \rightarrow \infty} r^{\alpha / \beta} f^{1 / m}(r ; \lambda)$ exists and finite. On the other hand, by (4.9),

$$
f(r) \geq f\left(r_{1}\right) r^{-d}, \quad r>r_{1} .
$$

These conflictions implies that $\mathrm{L}(\lambda)>0$. 
Remark 4.7. Obviously, the limit value $\mathrm{L}(\lambda)=0$ is achieved only when $f$ has the compact support and Proposition 4.3 and Theorem 4.6 remain true for the case $\alpha \leq \beta$.

We finally show that there exists a fast orbit.

Theorem 4.8. The set $\mathcal{S}_{2} \neq \emptyset$ and closed. Moreover, the interface relation holds

$\lim _{r \rightarrow R^{-}}\left(f^{[m(p-1)-1] /[m(p-1)]}\right)^{\prime}(r)=-[m(p-1)-1] /[m(p-1)] \beta^{1 /(p-1)} R^{1 /(p-1)}$

for any $\lambda \in \mathcal{S}_{2}$.

Proof. By Theorems 4.1 and 4.5, we immediately see that $\mathcal{S}_{2}$ is nonempty and closed set. From Lemma 2.2, any solution $f=f(r, \lambda)$ with $\lambda \in \mathcal{S}_{2}$ has a compact support, say, $[0, R]$ and $f$ satisfies condition $f(R)=0, f^{\prime}(R)=0$. Integrating the equation (1.10) from $r$ to $R$ we get

$$
\left|f^{\prime}\right|^{p-2} f^{\prime}(r)+\beta r f^{1 / m}(r)=(\alpha-\beta) \int_{r}^{R} f^{1 / m}(s) d s-f^{q / m}(r) .
$$

Dividing by $f$, we have

$$
\begin{aligned}
& \left.f^{\prime}\right|^{p-2} f^{\prime}(r) / f^{1 / m}(r)+\beta r \\
= & (\alpha-\beta) \int_{r}^{R} f^{1 / m}(s) d s / f^{1 / m}(r)-f^{(q-1) / m}(r) .
\end{aligned}
$$

Since $f$ is strictly decreasing, we find that

$$
0 \leq \int_{r}^{R} f^{1 / m}(s) d s \leq f^{1 / m}(r)(R-r) .
$$

Hence

$$
\lim _{r \rightarrow R^{-}} \int_{r}^{R} f^{1 / m}(s) d s / f^{1 / m}(r)=0 .
$$

Letting $r \rightarrow R^{-}$in (4.12) then we obtain

$$
\lim _{r \rightarrow R^{-}}\left|f^{\prime}\right|^{p-2} f^{\prime}(r) / f^{1 / m}(r)=-\beta R
$$

and which is equivalent to the second result of theorem.

In addition, we show the monotonicity of the solutions of the problem (1.10) with respect to $\lambda$ in the sense that two positive orbits do not intersect each other.

Theorem 4.9. Assume that $\alpha>0, \beta>0$ and $f_{i}$ are solutions of problem (1.10) on $\left[0, R_{i}\right)$ with initial data $f_{i}(0)=\lambda_{i}>0, i=1,2$, where $\left[0, R_{i}\right)$ denotes the maximal existence interval of $f_{i}$ and the $R_{i}$ are possibly infinity. Then

$$
\lambda_{2}>\lambda_{1} \Rightarrow f_{2}(r)>f_{1}(r) \text { for all } 0 \leq r \leq R:=\min \left\{R_{1}, R_{2}\right\} .
$$


Proof. Suppose contrarily that there exists $R_{0} \in[0, R]$ such that $f_{1}(r)<f_{2}(r)$ for $r \in\left[0, R_{0}\right)$ and $f_{1}\left(R_{0}\right)=f_{2}\left(R_{0}\right)$. We define

$$
g_{k}(r):=k^{-m p /[m(p-1)-1]} f_{1}(k r), \quad r \in\left[0, R_{1} / k\right)
$$

for $k>0$ and then $g_{k}(r)$ solves

$$
\begin{aligned}
& \left(\left|g_{k}^{\prime}\right|^{p-2} g_{k}^{\prime}\right)^{\prime}+\beta r\left(g_{k}^{1 / m}\right)^{\prime} \\
& \quad+\alpha g_{k}^{1 / m}+k^{[p q-(m+1)(p-1)] /[m(p-1)-1]}\left(g_{k}^{q / m}\right)^{\prime}=0 .
\end{aligned}
$$

By Lemma 2,1 we know that $f_{1}$ is strictly decreasing on $\left[0, R_{1}\right)$ and so $g_{k}$ is strictly decreasing with respect to $k$. In particular, $\lim _{k \rightarrow 0} g_{k}(r)=+\infty$ for any $r \in[0, R]$. Thus there exists a small $k_{0}>0$ such that

$$
g_{k}(r)>f_{2}(r) \text { for } r \in[0, R] \text { and } k \in\left[0, k_{0}\right]
$$

and the set

$$
I:=\left\{k \in\left(0, k_{0}\right) ; g_{k}(r)>f_{2}(r) \quad \text { for } r \in\left[0, R_{0}\right]\right\}
$$

is nonempty and open. Setting $l:=\sup I$, we see that $l<1, l \notin I$ and there exists $r_{0} \in\left[0, R_{0}\right]$ such that $g_{l}\left(r_{0}\right)=f_{2}\left(r_{0}\right)$.

If $r_{0}=R_{0}$, then $g_{l}\left(R_{0}\right)=l^{-m p /[m(p-1)-1]} f_{1}\left(l R_{0}\right)=f_{2}\left(R_{0}\right)$. Since $f_{1}\left(R_{0}\right)=$ $f_{2}\left(R_{0}\right)$ and $g_{l}$ is strictly decreasing with respect to $l$, we conclude that $l=1$ and which contradicts to the hypothesis. If $r_{0} \in\left(0, R_{0}\right)$, then $g_{l}$ much touch $f_{2}$ at $r=r_{0}$ from the above. But in this case we deduce from (1.10) that

$$
\begin{aligned}
& \left(\left|g_{l}^{\prime}\right|^{p-2} g_{l}^{\prime}\right)^{\prime \prime}\left(r_{0}\right)-\left(\left|f_{2}^{\prime}\right|^{p-2} f_{2}^{\prime}\right)^{\prime \prime}\left(r_{0}\right) \\
= & \left(1-l^{[p q-(m+1)(p-1)] /[m(p-1)-1]}\right)\left(f_{2}^{q / m}\right)^{\prime}\left(r_{0}\right)<0,
\end{aligned}
$$

which obviously violates the strong maximum principle. Thus $g_{l}$ much touch $f_{2}$ at $r=0$ from the above. But also from (1.10), we find $\left(\left|f_{2}^{\prime}\right|^{p-2} f_{2}^{\prime}\right)^{\prime}(0)=$ $-\alpha \lambda_{2}^{1 / m}$ and $\left(\left|f_{2}^{\prime}\right|^{p-2} f_{2}^{\prime}\right)^{\prime \prime}(0)=-\left(f_{2}^{q / m}\right)^{\prime \prime}(0)=-q / m \lambda_{2}^{q / m-1} f_{2}^{\prime \prime}(0)$. Similarly for $g_{l}$ and we obtain

$$
\begin{aligned}
& \left(\left|g_{l}^{\prime}\right|^{p-2} g_{l}^{\prime}\right)^{\prime \prime}(0)-\left(\left|f_{2}^{\prime}\right|^{p-2} f_{2}^{\prime}\right)^{\prime \prime}(0) \\
= & \left(l^{[p q-(m+1)(p-1)] /[m(p-1)-1]}-1\right) q / m \lambda_{2}^{q / m-1} f_{2}^{\prime \prime}(0)<0,
\end{aligned}
$$

which leads to another contradiction and completes all the proofs.

\section{Uniqueness}

In this section, we show that there exists only one fast decaying solution for the problem (1.10).

Recall that such a solution has compact support $[0, R]$ and has an interface relation

$$
\begin{aligned}
& \lim _{r \rightarrow R^{-}}\left(f^{[m(p-1)-1] /[m(p-1)]}\right)^{\prime}(r) \\
= & -[m(p-1)-1] /[m(p-1)] \beta^{1 /(p-1)} R^{1 /(p-1)}
\end{aligned}
$$

by Theorem 4.8 . 
Theorem 5.1. The set $\mathcal{S}_{2}$ consists only one element.

Proof. Let $F$ and $f$ be any two fast orbits with compact supports $\left[0, R_{i}\right]$ for $i=1,2$ respectively and satisfy $F(0)>f(0)$. We define

$$
f_{k}(r)=k f\left(k^{-\gamma} r\right), \quad \gamma=[m(p-1)-1] /(m p)
$$

and then $f_{k}$ will be larger than $F$ on $\left[0, R_{2}\right]$ for sufficiently large $k$. We now define

$$
\tau=\min \left\{k \geq 1 ; f_{k}(r) \geq F(r), 0 \leq r \leq R_{2}\right\} .
$$

The uniqueness proof is now reduced to showing that $\tau$ is not greater than 1 . Suppose that $\tau>1$, to the contrary. We will show that there exists $\epsilon>0$ such that $f_{\tau-\epsilon}(r) \geq F(r)$ for every $r \in\left[0, R_{2}\right]$. Indeed, we are going to show that $f_{\tau}(r)$ does not touch $F(r)$ in compact support $\left[0, R_{2}\right]$ by dividing into three cases;

(i) in the interior of the support,

(ii) at the origin,

(iii) at $R_{2}$.

In fact, $f_{\tau}(r)$ solves

$$
\begin{aligned}
& \left(\left|f_{\tau}^{\prime}\right|^{p-2} f_{\tau}^{\prime}\right)^{\prime}+\beta r\left(f_{\tau}^{1 / m}\right)^{\prime}+\alpha f_{\tau}^{1 / m}+\left(f_{\tau}^{q / m}\right)^{\prime} \\
= & -\tau\left(1-\tau^{q / m-\gamma-1}\right)\left(f^{q / m}\right)^{\prime} .
\end{aligned}
$$

(i) If $f_{\tau}$ touches $F$ at $r_{0} \in\left(0, R_{2}\right)$, then $f_{\tau}\left(r_{0}\right)=F\left(r_{0}\right), f_{\tau}^{\prime}\left(r_{0}\right)=F^{\prime}\left(r_{0}\right)<0$ and

$$
\left(\left|f_{\tau}^{\prime}\right|^{p-2} f_{\tau}^{\prime}\right)^{\prime}\left(r_{0}\right)<\left(\left|F^{\prime}\right|^{p-2} F^{\prime}\right)^{\prime}\left(r_{0}\right)
$$

But $f_{\tau}(r) \geq F(r)$ near $r=r_{0}$, which obviously violates the strong maximum principle.

(ii) If $f_{\tau}$ touches $F$ at $r_{0}=0$, then $f_{\tau}(0)=F(0)>0, f_{\tau}^{\prime}(0)=F^{\prime}(0)=0$ and $\left(\left|f_{\tau}^{\prime}\right|^{p-2} f_{\tau}^{\prime}\right)^{\prime}=-\alpha f_{\tau}^{1 / m}(0)=\left(\left|F^{\prime}\right|^{p-2} F^{\prime}\right)^{\prime}(0)<0$. Differentiating the equation (1.10) and (5.2), we reduce that

$$
\left(\left|f_{\tau}^{\prime}\right|^{p-2} f_{\tau}^{\prime}\right)^{\prime \prime}(0)-\left(\left|F^{\prime}\right|^{p-2} F^{\prime}\right)^{\prime \prime}(0)=-\tau\left(1-\tau^{q / m-\gamma-1}\right)\left(f^{q / m}\right)^{\prime \prime}(0)<0 .
$$

Thus, we have

$$
\left(\left|f_{\tau}^{\prime}\right|^{p-2} f_{\tau}^{\prime}\right)^{\prime \prime}(r)-\left(\left|F^{\prime}\right|^{p-2} F^{\prime}\right)^{\prime \prime}(r) \leq 0
$$

near $r_{0}=0$, which leads to a contradiction.

(iii) For the final case, we define the functions $u, U_{\tau}$ corresponding to $F$ and $f_{\tau}$ by

$$
\begin{gathered}
u(x, t)=: t^{-\alpha} F^{1 / m}(r), \\
U_{\tau}(x, t)=: t^{-\alpha} f_{\tau}^{1 / m}(r)=: \tau^{1 / m} t^{-\alpha} f^{1 / m}\left(\tau^{-\gamma} r\right),
\end{gathered}
$$

where $\gamma=(p-2) / p, r=r t^{-\beta}$ as defined before. Then $u(x, t)$ is a solution of (1.1) and $U_{\tau}$ is a supersolution. Indeed, a straightforward computation shows that

$U_{t}-\left(\left|\left(U^{m}\right)_{x}\right|^{p-2}\left(U^{m}\right)_{x}\right)_{x}-\left(U^{q}\right)_{x}=\tau^{1 / m}\left(\tau^{q / m-\gamma-1}-1\right)\left|\left(f^{q / m}\right)^{\prime}\right| \geq 0$ for $\tau>1$. 
Following directly the proof of Lemma 10 in [12], we can show that for fixed $t>0$ and all sufficiently small $\delta^{\prime}>0$, there exists $\theta=\theta\left(\delta^{\prime}\right) \in(0,1)$ such that $U_{\tau}(x, t) \leq U_{\tau}\left(x, t+\delta^{\prime}\right)$ if $x$ satisfies $\theta R_{2} \leq x t^{-\beta} \tau^{-\gamma} \leq R_{2}$ and $\lim _{\delta^{\prime} \downarrow 0} \theta\left(\delta^{\prime}\right)=\theta_{0} \in(0,1)$. In the proof, we use the interface relation (5.1) crucially (see $[12]$ for details). In particular, we have

$$
U_{\tau}(x, 1) \leq U_{\tau}\left(x, 1+\delta^{\prime}\right)
$$

for $\theta R_{2} \tau^{\gamma} \leq x<R_{2} \tau^{\gamma}\left(1+\delta^{\prime}\right)^{\beta}$. In other words, we found a separation near the right end $r=R_{2}$.

On the other hand, as previously proved, $f_{\tau}$ can not touches $F$ at $r_{0} \in$ $\left[0, R_{2}\right)$, which implies for any $\epsilon_{1}>0$, there exists $\kappa=\kappa\left(\epsilon_{1}\right) \in(0,1)$ such that $F^{1 / m}(x) \leq \kappa f_{\tau}^{1 / m}(x)$, that is

$$
u(x, 1) \leq \kappa U_{\tau}(x, 1) .
$$

We choose $\epsilon_{1}>0$ so that $0<\epsilon_{1}<1-\theta_{0}$ and find $\delta_{0}=\delta_{0}\left(\epsilon_{1}\right)$ such that

$$
1-\epsilon_{1}>\theta\left(\delta^{\prime}\right)
$$

for $\delta^{\prime} \in\left(0, \delta_{0}\right)$. By continuity of $U_{\tau}$, there exists $\delta_{1}=\delta_{1}\left(\epsilon_{1}\right) \in\left(0, \delta_{0}\right)$ such that

$$
\kappa U_{\tau}(x, 1) \leq U_{\tau}\left(x, 1+\delta^{\prime}\right)
$$

for any $\delta^{\prime} \in\left(0, \delta_{1}\right)$ and $0 \leq x<\left(1-\epsilon_{1}\right) R_{2} \tau^{\gamma}$. Combining (5.3), (5.4), and (5.6) and using again the continuity of $U_{\tau}$, we deduce that for $\delta \in\left(\delta^{\prime}, \delta_{1}\right), \delta-\delta^{\prime}$ small enough, we have

$$
F^{1 / m}(r)<U_{\tau}(x, 1+\delta)=\tau^{1 / m}(1+\delta)^{-\alpha} f^{1 / m}\left(x(1+\delta)^{-\beta} \tau^{-\gamma}\right)
$$

for any $x \geq 0$. Furthermore, from the continuity with respect to $\tau$, there exists $\tau_{1} \in(0, \tau)$ such that

$$
\begin{aligned}
u(x, 1) & =F^{1 / m}(r) \leq \tau_{1}^{1 / m}(1+\delta)^{-\alpha} f^{1 / m}\left(x(1+\delta)^{-\beta} \tau_{1}^{-\gamma}\right) \\
& =U_{\tau_{1}}(x, 1+\delta)
\end{aligned}
$$

for any $x \geq 0$. By parabolic maximum principle, we have $u(x, t) \leq U_{\tau_{1}}(x, t+\delta)$, that is,

$$
t^{-\alpha} F^{1 / m}\left(x t^{-\beta}\right) \leq \tau_{1}^{1 / m}(t+\delta)^{-\alpha} f^{1 / m}\left(x(t+\delta)^{-\beta} \tau_{1}^{-\gamma}\right)
$$

for any $t \geq 1$ and $x \geq 0$. Rewriting (5.8) of the form;

$$
F^{1 / m}(r) \leq \tau_{1}^{1 / m}[t /(t+\delta)]^{-\alpha} f^{1 / m}\left(r[t /(t+\delta)]^{-\beta} \tau_{1}^{-\gamma}\right)
$$

and letting $t \longrightarrow \infty$, we find that

$$
F^{1 / m}(r) \leq \tau_{1}^{1 / m} f^{1 / m}\left(r \tau_{1}^{-\gamma}\right)
$$

which contradicts the fact that $\tau$ is the smallest constant with that property. Thus $f_{\tau}$ does not meet at $r_{0}=R_{2}$.

Hence we may find $\epsilon>0$ so that

$$
f_{\tau-\epsilon}(r) \geq F(r) \quad \text { for every } r \in\left[0, R_{2}\right]
$$


which means that we can slightly reduce the factor $\tau$. Hence we may conclude that $\tau=1$ but it is obviously impossible.

\section{References}

[1] J. S. Baek, M. Kwak, and K. Yu, Uniqueness of the very singular solution of a degenerate parabolic equation, Nonlinear Anal. 45 (2001), no. 1, Ser. A: Theory Methods, 123-135.

[2] P. Biler and G. Karch, A Neumann problem for a convection-diffusion equation on the half-line, Ann. Polon. Math. 74 (2000), 79-95.

[3] H. Brezis, L. A. Peletier, and D. Terman, A very singular solution of the heat equation with absorption, Arch. Rational Mech. Anal. 95 (1986), no. 3, 185-209.

[4] J. L. Diaz and J. E. Saá, Uniqueness of very singular self-similar solution of a quasilinear degenerate parabolic equation with absorption, Publ. Mat. 36 (1992), no. 1, 19-38.

[5] E. DiBenedetto, Degenerate Parabolic Equations, Springer-Verlag, New York, 1993.

[6] M. Escobedo, O. Kavian, and H. Matano, Large time behavior of solutions of a dissipative semilinear heat equation, Comm. Partial Differential Equations 20 (1995), no. 7-8, $1427-1452$.

[7] M. Escobedo and E. Zuazua, Large time behavior for convection-diffusion equations in $R^{N}$, J. Funct. Anal. 100 (1991), no. 1, 119-161.

[8] M. Escobedo, J. L. Vázquez, and E. Zuazua, A diffusion-convection equation in several space dimensions, Indiana Univ. Math. J. 42 (1993), no. 4, 1413-1440.

[9] L. C. Evans, Partial Differential Equations, American Mathematical Society, Providence, RI, 1998.

[10] Z. B. Fang and M. Kwak, Complete classification of shape functions of self-similar solutions, J. Math. Anal. Appl. 330 (2007), no. 2, 1447-1464.

[11] M. Guedda, Self-similar solutions to a convection-diffusion processes, Electron. J. Qual. Theory Differ. Equ. 2000 (2000), no. 3, 17 pp.

[12] S. Kamin and L. Veron, Existence and uniqueness of the very singular solution of the porous media equation with absorption, J. Analyse Math. 51 (1988), 245-258.

[13] M. Kwak, A semilinear heat equation with singular initial data, Proc. Roy. Soc. Edinburgh Sect. A 128 (1998), no. 4, 745-758.

[14] - A porous media equation with absorption. II. Uniqueness of the very singular solution, J. Math. Anal. Appl. 223 (1998), no. 1, 111-125.

[15] _ A porous media equation with absorption. I. Long time behaviour, J. Math. Anal. Appl. 223 (1998), no. 1, 96-110.

[16] M. Kwak and K. Yu, Asymptotic behaviour of solutions of a degenerate parabolic equation, Nonlinear Anal. 45 (2001), no. 1, Ser. A: Theory Methods, 109-121.

[17] $\mathrm{Ph}$. Laurençot and F. Simondon, Source-type solutions to porous medium equations with convection, Commun. Appl. Anal. 1 (1997), no. 4, 489-502.

[18] _ Long-time behaviour for porous medium equations with convection, Proc. Roy. Soc. Edinburgh Sect. A 128 (1998), no. 2, 315-336.

[19] G. Leoni, On the existence of fast-decay solutions for a quasilinear elliptic equation with a gradient term, Atti Sem. Mat. Fis. Univ. Modena 46 (1998), suppl., 827-846.

[20] L. A. Peletier and H. C. Serafini, A very singular solution and other self-similar solutions of the heat equation with convection, Nonlinear Anal. 24 (1995), no. 1, 29-49.

[21] L. A. Peletier and D. Terman, A very singular solution of the porous media equation with absorption, J. Differential Equations 65 (1986), no. 3, 396-410.

[22] L. A. Peletier and J. Wang, A very singular solution of a quasilinear degenerate diffusion equation with absorption, Trans. Amer. Math. Soc. 307 (1988), no. 2, 813-826.

[23] Z. Q. Wu, J. N. Zhao, J. X. Yin, and H. L. Li, Nonlinear Diffusion Equations, World Scientific Publishing Co., Inc., River Edge, NJ, 2001. 
[24] J. N. Zhao, The asymptotic behaviour of solutions of a quasilinear degenerate parabolic equation, J. Differential Equations 102 (1993), no. 1, 33-52.

School of Mathematical Sciences

Ocean University of China

QINGDAO, 266-071, P. R. China

E-mail address: fangzb7777@hotmail.com 